\title{
On the origin of white dwarfs with carbon-dominated atmospheres: the case of H1504+65
}

\author{
L. G. Althaus ${ }^{1,2,3}$, A. H. Córsico ${ }^{1,2,3}$, S. Torres ${ }^{4,5}$, and E. García-Berro ${ }^{4,5}$ \\ ${ }^{1}$ Facultad de Ciencias Astronómicas y Geofísicas, Universidad Nacional de La Plata, Paseo del Bosque S/N, (1900) La Plata, \\ Argentina \\ e-mail: [althaus; acorsico]@fcaglp.unlp.edu.ar \\ 2 Instituto de Astrofísica La Plata, IALP, CONICET-UNLP, Argentina \\ 3 Member of the Carrera del Investigador Científico y Tecnológico, CONICET, Argentina \\ 4 Departament de Física Aplicada, Escola Politècnica Superior de Castelldefels, Universitat Politècnica de Catalunya, \\ Av. del Canal Olímpic, s/n, 08860 Castelldefels, Spain \\ e-mail: [santi;garcia]@fa.upc.edu \\ 5 Institute for Space Studies of Catalonia, c/Gran Capità 2-4, Edif. Nexus 104, 08034 Barcelona, Spain
}

Received 6 August 2008 / Accepted 12 November 2008

ABSTRACT

\begin{abstract}
Aims. We explore different evolutionary scenarios to explain the helium deficiency observed in H1504+65, the most massive known PG1159 star.

Methods. We concentrate mainly on the possibility that this star could be the result of mass loss shortly after its born-again and during its subsequent evolution through the [WCL] stage. This possibility is consistent with observational evidence of extensive mass-loss events in Sakurai's object and is in line with the finding that these mass losses give rise to PG1159 models with thin heliumrich envelopes and high rates of period change, as demanded by the pulsating star PG1159-035. We compute the post-born-again evolution of massive sequences by taking into account different mass-loss rate histories.

Results. Our results show that stationary winds during the post-born-again evolution fail to remove completely the helium-rich envelope and are therefore unable to explain the helium deficiency observed in H1504+65. Stationary winds during the Sakurai and [WCL] stages remove at most half of the envelope that survives the violent hydrogen burning during the born-again phase.

Conclusions. In view of our results, the proposed evolutionary sequence of born-again stars to H1504+65 and then to white dwarfs with carbon-rich atmospheres is difficult to reproduce unless the entire helium-rich envelope is ejected by non-stationary mass-loss episodes during the Sakurai stage.
\end{abstract}

Key words. stars: interiors - stars: evolution - stars: white dwarfs - stars: AGB and post-AGB

\section{Introduction}

Hydrogen (H)-deficient PG1159 stars are a transition stage between the post-asymptotic giant branch (AGB) stars and most of the H-deficient white dwarfs. Spectroscopic analyses reveal that PG1159 stars are characterized by a peculiar surface chemical composition, with most of them exhibiting helium (He)-, carbon- and oxygen-rich abundances at their surfaces. Inferred surface mass abundances are typically $X_{\mathrm{He}} \simeq 0.33, X_{\mathrm{C}} \simeq 0.5$, and $X_{\mathrm{O}} \simeq 0.17$, though notable variations are found between stars (Dreizler \& Heber 1998; Werner 2001). Currently, about 40 stars are members of the PG1159 spectroscopic family (Werner \& Herwig 2006), which covers a wide range of surface gravities $-5.5 \lesssim \log g \lesssim 8$ (in cgs units) - and effective temperatures $75000 \mathrm{~K} \lesssim T_{\text {eff }} \lesssim 200000 \mathrm{~K}$. PG1159 stars are thought to be formed as a result of a born-again episode, that is, a very late thermal pulse (VLTP) experienced by a hot white dwarf during its early cooling phase (see Schönberner 1979; Iben et al. 1983, for early references) or a late thermal pulse that occurs during the post-AGB evolution when $\mathrm{H}$ burning is still active (Blöcker 2001).

The variety of surface compositions observed in most PG1159 stars has been successfully explained by detailed simulations of the evolutionary stages prior to their formation, particularly the born-again episode (Werner \& Herwig 2006; Rauch 2006). For instance, the high oxygen abundance detected in the atmospheres of these stars and the presence of neon lines in the optical spectra of some PG1159 stars is in line with improved evolutionary calculations of the born-again scenario that incorporate convective overshoot (Herwig et al. 1999). However, some remaining cases still represent a challenge to the stellar evolution theory, which is unable to explain their origin adequately. This is true for the enigmatic star $\mathrm{H} 1504+65$, whose effective temperature of $T_{\text {eff }}=200000 \mathrm{~K}$ and high mass - between 0.74 and $0.97 M_{\odot}$ (Werner \& Herwig 2006; Werner et al. 2008) - make it the hottest and most massive known PG1159 star. In particular, H1504+65 is a H- and He-deficient star, and its atmosphere is dominated by carbon and oxygen plus traces of heavier elements $-X_{\mathrm{C}} \simeq 0.48, X_{\mathrm{O}} \simeq 0.48, X_{\mathrm{Ne}} \simeq 0.02, X_{\mathrm{Mg}} \simeq 0.02$ (Werner et al. 2004). It appears as if we are observing the naked carbon-oxygen core of this star. These unique characteristics of the known PG1159-type stars make the evolutionary history of H1504+65 very intriguing.

The discovery of a cool white-dwarf population with carbonrich atmospheres (Dufour et al. 2007, 2008) has renewed the interest in H1504+65 since it opens the possibility that H1504+65 could be the progenitor of this white-dwarf population. However, there is no yet a clear consensus about the evolutionary history 
that could explain the origin of its He deficiency. In this paper, we explore different evolutionary scenarios to explain the origin of H1504+65. Most importantly, we assess the viability that mass loss during the evolutionary stages following the VLTP when the star becomes a red gaint (Sakurai's stage) and the following [WCL] stage could be responsible for the He deficiency observed in H1504+65. This is an attractive idea in view of the finding by Althaus et al. (2008) that PG 1159 stars could be characterized by He-rich envelopes markedly smaller than those predicted by the standard theory of stellar evolution for the formation of PG1159 stars (Herwig et al. 1999; Miller Bertolami \& Althaus 2006).

\section{Proposed evolutionary scenarios}

It has been speculated that H1504+65 could have an evolutionary history completely different from that of the standard PG1159 stars. In particular, it has been suggested - and discarded - that H1504+65 could be a normal H-rich post-AGB star in which strong mass loss could remove the He-rich intershell (Schönberner \& Blöcker 1992). For this to be possible, very high mass-loss rates - of about $10^{-7} M_{\odot} / \mathrm{yr}$ - should occur at low luminosities. However, it is dubious that such mass-loss rates could be maintained by real stars during the white dwarf stage. It has also been suggested (Werner 2001) that H1504+65 could be the result of the evolution of a heavy-weight, intermediatemass star of initial mass between 9 and $11 M_{\odot}$ that has burned carbon into oxygen and neon (Ritossa et al. 1996; García-Berro et al. 1997). In this scenario, He would be burned during a late carbon-shell flash in the former Super-AGB phase, although detailed simulations do not appear to support this idea. Instead, the theoretical calculations predict that carbon flashes cease before the thermally-pulsing AGB phase is reached (Ritossa et al. 1996). In this scenario, the massive envelope of these stars should be lost before reaching the thermally pulsing AGB phase, which would require unrealistic mass-loss rates during the early AGB phase. In addition, the resulting oxygen-neon core would be too massive $\left(\sim 1.1 M_{\odot}\right)$ to be consistent with both the mass of H1504+65 and the spectral features observed in the carbon-rich, white-dwarf population detected by Dufour et al. (2007, 2008). Another possibility would be that H1504+65 is the product of the coalescence of two typical carbon-oxygen white dwarfs (Guerrero et al. 2004). However, given the short age of $\mathrm{H} 1504+65$, this possibility appears unlikely. If this were the case, this star should be rapidly spinning and, moreover, the debris of the disrupted secondary should form a thick, massive, keplerian disk around H1504+65, which is not observed.

A different possibility is that $\mathrm{H} 1504+65$ has an evolutionary origin that does not differ significantly from those of the majority of PG1159 stars; namely that its progenitor has experienced a born-again episode. Werner et al. (2004) raised the possibility that, because of the high mass of this star, He has been completely burned into carbon and oxygen during the late He-shell flash. Detailed simulations of the born-again stage for massive remnants (Miller Bertolami \& Althaus 2006) illustrate, however, that this scenario is impossible, since our calculations show that even for the most massive born-again sequence (of mass $0.87 M_{\odot}$ ), most of the He content before the born-again episode remains unburnt. This is an expected result because He-burning during a late He flash does not differ from the well-studied cases of thermal pulses on the AGB, for which the available calculations do not exhibit in any case a complete depletion of the intershell He during the thermal pulse.
Table 1. Main characteristics of the post-born-again remnants.

\begin{tabular}{ccc}
\hline \hline $\bar{M} / M_{\odot}$ & $M_{\mathrm{He}} / M_{\odot}$ & $\log \left(L_{\max } / L_{\odot}\right)$ \\
\hline 0.561 & 0.040 & 3.94 \\
0.564 & 0.037 & 3.95 \\
0.664 & 0.021 & 4.17 \\
0.741 & 0.014 & 4.32 \\
0.870 & 0.0058 & 4.58 \\
\hline
\end{tabular}

An alternative possibility, which we explore in detail below, is that H1504+65 is the result of mass loss during the Sakurai's and [WCL] stages. The possibility that a large fraction of the He envelope could be lost during the Sakurai's stage is attractive and is supported by independent pieces of evidence. The first is that V4334 Sgr (Sakurai's object) exhibits significant mass-loss events (van Hoof et al. 2007). The second piece of evidence is the theoretical finding (Althaus et al. 2008) that, as a consequence of these high mass-loss rates during the Sakurai stage, PG1159 stars could be characterized by much thinner He envelopes than traditionally thought. As demonstrated in Althaus et al. (2008), a thin He-rich envelope appears to be needed to solve the longstanding discrepancy between the observed (see e.g. Costa \& Kepler 2008, for measurements) and theoretical rates of period change in the pulsating star PG1159-035.

\section{Post-born-again evolutionary sequences}

Our evolutionary calculations were completed using the LPCODE evolutionary code (Althaus et al. 2005), which computes the formation and evolution of white dwarfs through late thermal pulses, on the basis of a detailed description of the physical processes relevant to the calculation of the violent proton-burning phase during the born-again stage, particularly diffusive overshooting and non-instantaneous mixing. The code was also used by Miller Bertolami \& Althaus (2006), who computed a full set of PG1159 evolutionary sequences from ZAMS progenitors with initial masses ranging from 1.0 to $5.5 M_{\odot}$ to the born-again stage. In particular, the $0.87 M_{\odot}$ model sequence, the most massive of the computed sequences, is the result of the full evolution of an initially $5.5 M_{\odot}$ main sequence model star.

In Table 1, we show, for each stellar mass, the He envelope mass of our models remaining shortly after the occurrence of the born-again episode - that is, by the time the Sakurai's stage is reached. We define the He envelope as the region of the star where the abundance mass fraction of $\mathrm{He}$ is higher than 0.01 . We also list the luminosity achieved shortly after the Sakurai stage. Note that for these sequences, which cover most of the observed mass range of PG1159 stars, the expected range of He envelope masses spans almost one order of magnitude. These values are upper limits because no mass loss was introduced in our evolutionary sequences after the occurrence of the VLTP. We also note that the most massive models are characterized by He envelopes whose masses are substantially lower than those of the less massive models. In particular, for the sequence with $0.87 M_{\odot}$ - representative of the spectroscopic mass of H1504+65 (Werner \& Herwig 2006; Miller Bertolami \& Althaus 2006) - the He envelope mass remaining amounts to only $\sim 5.8 \times 10^{-3} M_{\odot}$. Finally, it is also noted the significant increase in the maximum luminosities with increasing mass. 


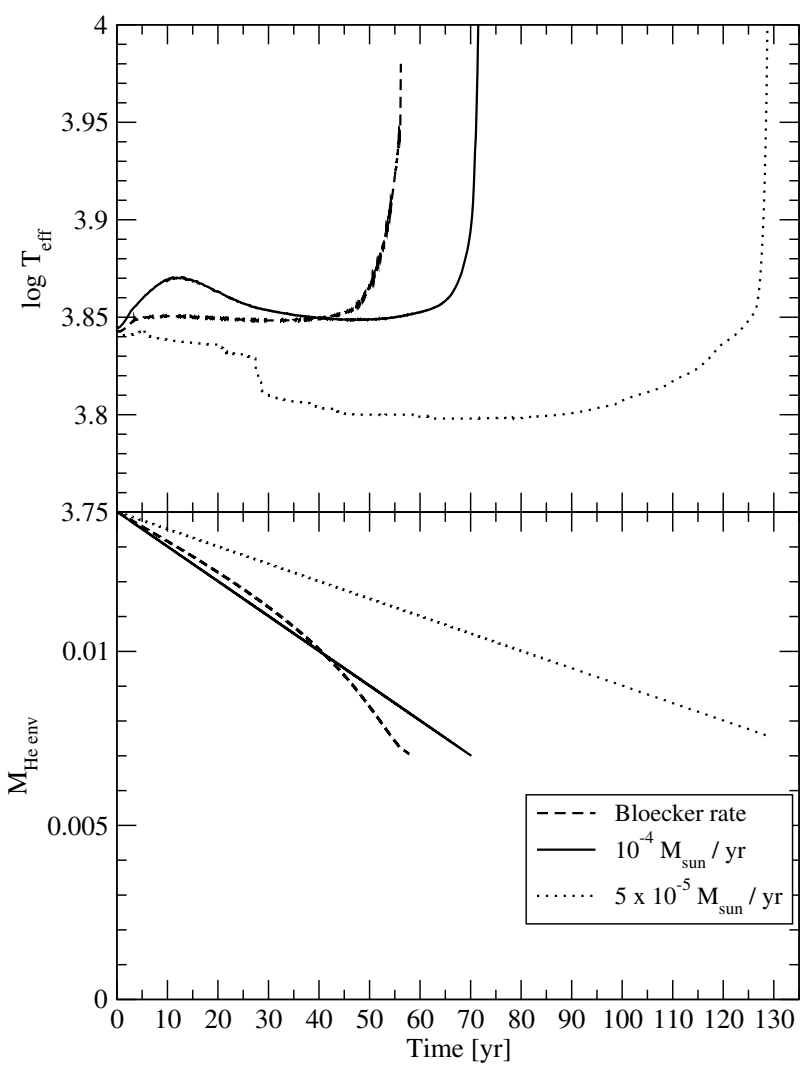

Fig. 1. The effective temperature and mass of the He envelope (in solar masses) of the $0.741 M_{\odot}$ model sequence as a function of time during the Sakurai stage. Different mass-loss rates as indicated in the legend have been assumed.

\section{A post-born-again origin for $\mathrm{H} 1504+65$ ?}

The fact that the most massive models are characterized by a small He-rich envelope is particularly attractive in view of the evidence presented by Van Hoof et al. (2007) about strong mass losses in Sakurai's object, which has a mass of about $0.60 M_{\odot}$. We note that if the total mass lost during the Sakurai stage is as high as $0.005 M_{\odot}$, which is not discarded by new radio and optical observations of the Sakurai's object (Van Hoof et al. 2007), then post-born-again remnants as massive as about $0.9 M_{\odot}$ are, in principle, expected to lose their entire He-rich envelope, exposing the carbon-oxygen core during their subsequent evolution to the PG1159 regime (see Table 1). To explore this possibility, we computed the evolution of the 0.741 and $0.87 M_{\odot}$ sequences from the born-again stage to the PG1 159 phase by assuming stationary mass losses during the Sakurai and [WCL] stages. For the Sakurai phase, we considered the mass-loss rate of Blöcker (1995) and two constant mass-loss rates of $\dot{M}=5 \times 10^{-5} M_{\odot} / \mathrm{yr}$ and $M=10^{-4} M_{\odot} /$ yr. During the [WCL] stage high mass losses have also been reported by Koesterke (2001). During this phase mass loss depends strongly on the surface luminosity of the remnant. To account for this, we adopted the mass-loss rate of Lawlor \& MacDonald (2006). In particular, mass-loss rates of $\sim 10^{-5} M_{\odot} /$ yr are expected for a [WCL] star with $\log \left(L / L_{\odot}\right) \gtrsim$ 4.5 .

Irrespective of the mass-loss rate during the Sakurai stage, our calculations show that the mass of the final He envelope is the same independent of the mass-loss rate prescription. Indeed, only about half the total mass of the He-rich envelope can be eroded during this stage. This is illustrated in Fig. 1 for the case of $0.741 M_{\odot}$ sequence. During this stage and despite the distinct

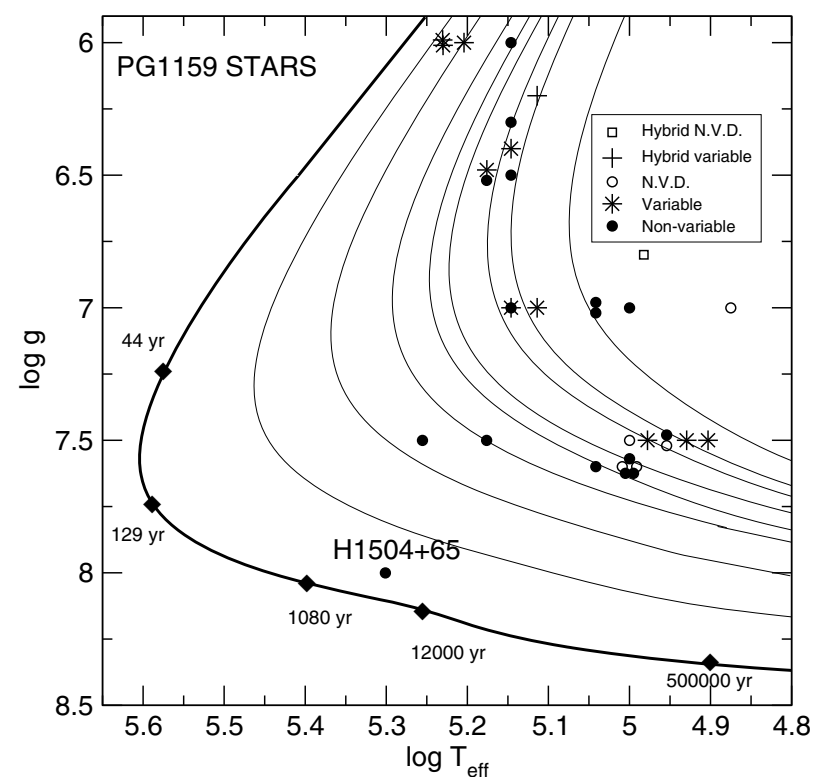

Fig. 2. Surface gravity versus effective temperature for the evolutionary sequences of Miller Bertolami \& Althaus (2006) with different stellar masses - thin lines $(0.741,0.664,0.609,0.589,0.565,0.542,0.53$, and $0.51 M_{\odot}$, from left to right) - and the $0.87 M_{\odot}$ model sequence with thin He-rich envelope - thick line. For the latter, post-born-again ages are labelled along the curve. The mass-loss rates of Lawlor \& MacDonald (2006) have been adopted during the [WCL] stage. The available observational data for selected PG 1159 stars are also included. Spectroscopic uncertainties are typically 0.5 dex for the gravity and between 10 and $15 \%$ for the effective temperature.

evolutionary timescales resulting from the different mass-loss prescriptions, the final mass of the He-rich envelope is only reduced from 0.014 to about $0.007 M_{\odot}$. Below a critical $T_{\text {eff }}$, mass loss is no longer efficient in removing the He envelope. In particular, we note that because of the higher mass loss provided by the Blöcker (1995) formulation, departure from the Sakurai stage in this case occurs earlier. We also found that as soon as the envelope mass is reduced below the critical value of about $0.007 M_{\odot}$, in the case of our $0.741 M_{\odot}$ sequence, the subsequent evolution through the [WCL] stage proceeds extremely rapidly, in a few decades for the $0.87 M_{\odot}$ sequence (see Fig. 2). As a result of this rapid evolution, the mass lost from the surviving He-rich envelope during the [WCL] stage amounts to only a few $10^{-4} M_{\odot}$, independently of the exact amount of mass lost during the Sakurai stage. This is much smaller than the final He-rich envelope mass with which the star departs from its Sakurai stage. We conclude that stationary winds during the post-born-again evolution fail to remove the entire He-rich envelope and cannot explain the observed He deficiency of H1504+65. In other words, the residual He-rich envelope could only be eroded during the [WCL] stage as long as most of the envelope is lost during the Sakurai stage, which, according to our calculations, is impossible if stationary winds are assumed. Conversely, if the entire He-rich envelope is to be removed, non-stationary, mass-loss episodes during the Sakurai stage are required.

\section{Conclusions and discussion}

The evidence presented in this investigation suggests that mass losses during the Sakurai stage could produce PG1159 stars characterized by He envelopes substantially thinner than those traditionally accepted by the standard theory of stellar evolution 
(Herwig 1999; Miller Bertolami \& Althaus 2006). Motivated by the less massive He envelope remaining after the prior evolution of massive born-again stars, we have explored the possibility that these stars may lose almost all of their residual envelopes as a result of stationary mass-loss. To this end, we have computed the full evolution of massive post-born-again stars by considering a variable mass-loss history as well as constant mass-loss rates during the Sakurai stage. We found that stationary winds during the post-born-again evolution do not completely remove the He-rich envelope and, thus, cannot explain the absence of $\mathrm{He}$ in H1504+65. Stationary mass-losses during the Sakurai stage remove at most a half of the He-rich envelope surviving the violent $\mathrm{H}$ burning during the born-again phase, and the following evolution during the [WCL] stage proceeds too rapidly to erode the remaining envelope with [WCL] winds. This result is independent of the detailed treatment of mass loss during the Sakurai stage. Thus, the origin of the white-dwarf population discovered to have carbon-dominated atmospheres cannot be traced back to VLTP progenitors that have undergone stationary mass-loss during their Sakurai stage. Conversely, for H1504+65 to represent the evolutionary link between born-again stars and white dwarfs with carbon-rich atmospheres, most of the He-rich envelope should be ejected by non-stationary winds during the Sakurai stage. This possibility is not entirely discarded since ejection of the entire He-rich envelope should occur from the layers at which the thermal energy input of the He flash occurs and above which our evolutionary calculations show that the envelope rapidly expands. The feasibility that most of the envelope could be ejected in more massive models is strengthened by the fact that a massive remnant is characterized by a markedly higher surface luminosity (by a factor of 4) and a higher input energy from the He flash, than Sakurai's object. In closing, we note that the possibility that traces of He remain in H1504+65 cannot be totally discarded. If some He survives the mass-loss events, diffusion will lead to a He-rich white dwarf and the carbon-rich atmosphere should emerge as a result of convective dredge-up at lower effective temperatures. Exploring this possibility requires a more elaborate treatment of evolution during the Sakurai stage and is beyond the scope of the present work.

Acknowledgements. We acknowledge useful discussions with M. M. Miller Bertolami. Part of this work was supported by the MEC grant AYA05-08013C03-01, by the European Union FEDER funds, by the AGAUR, by AGENCIA through the Programa de Modernización Tecnológica BID 1728/OC-AR, and by PIP 6521 grant from CONICET.

\section{References}

Althaus, L. G., Córsico, A. H., Miller Bertolami, M. M., García-Berro, E., \& Kepler, S. O. 2008, ApJ, 677, L35

Althaus, L. G., Serenelli, A. M., Panei, J. A., et al. 2005, A\&A, 435, 631

Blöcker, T. 1995, A\&A, 297, 727

Blöcker, T. 2001, Ap\&SS, 275, 1

Costa, J. E. S., \& Kepler, S. O. 2008, A\&A, 489, 1225

Dreizler, S., \& Heber, U. 1998, A\&A, 334, 618

Dufour, P., Liebert, J., Fontaine, G., \& Behara, N. 2007, Nature, 450, 522

Dufour, P., Fontaine, G., Liebert, J., Schmidt, G.D., \& Behara, N. 2008, ApJ, 683,978

García-Berro, E., Ritossa, C., \& Iben, I. 1997, ApJ, 485, 765

Guerrero, J., García-Berro, E., \& Isern, J. 2004, A\&A, 257

Herwig, F., Blöcker, T., Langer N., \& Driebe, T. 1999, A\&A, 349, L5

Iben, I., Kaler, J. B., Truran, J. W., \& Renzini, A. 1983, ApJ, 264, 605

Koesterke, L. 2001, Ap\&SS, 275, 41

Lawlor T. M., \& MacDonald J. 2006, MNRAS, 371, 236

Miller Bertolami, M. M., \& Althaus, L. G. 2006, A\&A, 454, 845

Rauch, T. 2006, in Planetary Nebulae in our Galaxy and Beyond, IAU Symp., 234, 131, in press [arXiv: $0711.4565 \mathrm{v} 1]$

Ritossa, C., García-Berro, E., \& Iben, I. 1996, ApJ, 460, 489

Schönberner, D. 1979, A\&A, 79, 108

Schönberner, D., \& Blöcker, T. 1992, in The atmospheres of Early-Type Stars, Lectures Notes in Physics (Springer), 401, 305

Van Hoof, P. A. M., Hajduk, M., Zijlistra A. A., et al. 2007, A\&A, 471, L9

Werner, K. 2001, Ap\&SS, 275, 27

Werner, K., \& Herwig, F. 2006, PASP, 118, 183

Werner, K., Rauch, T., Barstow, M. A., \& Kruk, J. W. 2004, A\&A, 421, 1169

Werner, K., Rauch, T., Reiff, E., \& Kruk, J. W. 2008, in Hydrogen-deficient stars, ASP Conf. Ser., 391, 109 